\title{
The properties of children's language are caused by those of their mothers', and vice-versa
}

Fermín Moscoso del Prado Martín ${ }^{* 1}$, Jeremy Irvin ${ }^{2,3} \&$ Daniel Spokoyny ${ }^{2}$

${ }^{1}$ Department of Linguistics, University of California, Santa Barbara

${ }^{2}$ College of Creative Studies, University of California, Santa Barbara

${ }^{3}$ Department of Computer Science, Stanford University

Both the language used by young children (child language; CL) and the simplified language used by caretakers when talking to them (child-directed speech; $\operatorname{CDS}^{1}$ ) become increasingly complex along development, eventually approaching regular adult language ${ }^{2,3}$. Researchers disagree on whether children learn grammar from the input they receive (usage-based theories $\left.^{4-6}\right)$, or grammars are mostly innate, requiring only minimal input-based adjustments on the part of the children (nativist theories ${ }^{7,8}$ ). A related question is whether parents adapt the complexity of CDS in specific response to their children's language abilities ${ }^{3,9-11}$, or only in response to their level of general cognitive development ${ }^{12-14}$. Parent-child interactions can be modelled by nonlinear dynamical systems ${ }^{15-17}$. A technique recently developed in Ecology, Convergent Cross-Mapping (CCM) ${ }^{18,19}$, enables assessing causal relations between series from a nonlinear dynamical system. Here, we use CCM to reconstruct the network of causal relations between aspects of $\mathrm{CL}$ and CDS. This network supports a lexically-based syntax that is statistically learned. The results also indicate that mothers adapt CDS in response mainly to the grammar of CL. Our findings verify the strong causal predictions of usage-based theories ${ }^{4-6}$, and are difficult for nativist theories ${ }^{7,8}$ to account for. 
Linguistics distinguishes between lexical (i.e., which words are used), morphological (i.e., which variants of the words are used, as in singular/plural, etc.), and syntactic (i.e., how the words are combined) aspects of human language. There is broad agreement in that lexical aspects of language are learned by children. However, when it comes to grammatical (ie., morphological and syntactic) aspects, researchers coming from the Generative Linguistics tradition argue that experience plays only a minimal role in the acquisition of grammar; instead children would rely mostly on innate grammatical knowledge, requiring only adjustments for each specific language ${ }^{7,8}$. Others propose usage-based theories of acquisition, in which morphology and syntax, rather than being innate, are also learned from the input ${ }^{4-6}$. In the latter views, children acquire different aspects at different paces, making use of statistical regularities that enable generalizations within and across the lexical and grammatical levels ${ }^{4-6}$. Children can generalize syntactic structures only after a sufficient number of words have been learned ${ }^{20}$, in what is termed the lexical basis of syntax $^{4-6,21}$. Conversely, syntactic bootstrapping ${ }^{22}$ indicates syntactic knowledge helps children in inferring the meanings of verbs and abstract nouns. From a usage-based perspective, the temporally increasing complexity of a child's input (CDS) serves the functional role of facilitating the task of language acquisition ${ }^{2,3,9-11}$. Consequently, the fine-tuning hypothesis ${ }^{10}$ proposes that mothers adapt the complexity of their language to their children's linguistic abilities at that point in time. These views are questioned by those claiming that the relative simplicity of CDS cannot be directly tied to the specific properties of a child's language ${ }^{8,12-14}$. In contrast, that grammar is learned also implies that the grammars learned by children will vary as a function of the CDS they have experienced, leading to individual differences ${ }^{23,24}$. 
Notice that the usage-based theories above make testable causal predictions: The lexical basis of syntax predicts that increases in the lexical diversity of CDS -by providing sufficient examples for generalizing syntactic structures- should cause increases in the syntactic diversity of CL. In turn, syntactic bootstrapping predicts that the lexical diversity of CL causally depends on its syntactic diversity, because the some words' meanings depend on prior understanding of relevant syntactic structures. Most crucially, fine-tuning predicts that changes in CL should cause changes in CDS, reflecting how parents adjust their language to the specific linguistic performance of their children. Previous research has found temporal lags between lexical and syntactic development ${ }^{20}$, as well as correlations between specific measures of a mother's CDS and her child's $\mathrm{CL}^{10,11,23,24}$. Although both of these findings are consistent with the causal predictions above, neither by itself constitutes explicit evidence for actual causality. The temporal lags could well be due to the delayed effects of a common causal variable (such as the child's overall level of cognitive development), and the symmetrical nature of the correlations makes it impossible to assess directional (causal) relations.

Dynamical systems offer powerful tools for modeling human development across many timescales $^{15}$. In human development, such systems extend beyond the individual: The linguistic and behavioral interactions between parents and children form a single dynamical system, jointly encompassing the child and the parent ${ }^{15-17}$. One difficulty that arises when trying to model a dynamical system as complex as the development of language in a parent-child dyad is that many factors that are important for the evolution of the system may not be available or there may be additional variables relevant for the system of which the modeller is not even aware. A crucial 
development towards overcoming this problem comes from the discovery that, in a deterministic coupled dynamical system, the dynamics of the whole system can be satisfactorily recovered using measurements of just a single of the system's variables using time-delayed embeddings (cf., Takens' Theorem ${ }^{25}$ ). Such reconstructions are possible even in situations with a substantial component of noise $\mathrm{e}^{26}$. Takens Theorem entails that, if a series $x$ influences another $y$, the historical values of $x$ can be predicted from those of $y$ (i.e., cross-mapping), and the quality of the predictions should increase with the number of points from $y$ 's embedding used for predicting $x$ (i.e., convergence). The CCM technique ${ }^{18}$ tests these properties by building a time-delayed embedding of $y$ and using it for estimating the values of $x$. The causal effect of $x$ on $y$ is then measured by the converging accuracy of $x$ 's predicted values. Crucially, CCM distinguishes the cases of variables being causally related, from the spurious causality that might appear when a third unknown variable is driving both variables of interests ${ }^{18}$, even in the presence of substantial noise ${ }^{27}$. The multispatial CCM method (mCCM) ${ }^{19}$ extends the causality tests to sets of multiple short time series measured on different instances of similar systems. Across languages and cultures, language acquisition is known to proceed through a series of stages $^{30}$ and events, which occur roughly at the same ages ${ }^{4-7,30}$ for most children (e.g., babbling, holophrastic stage, vocabulary burst, two-word stage, ... ). This can be modelled as the result of a growth process shared across children ${ }^{15}$. This enables taking sequences of linguistic development measurements from different children as trajectories of similar dynamical systems, thereby introducing the potential to employ $\mathrm{mCCM}$ to investigate language acquisition.

We analysed longitudinal samples of CL and CDS of twelve English-speaking children and 
their mothers ${ }^{28}$. At each time-point, we computed -for each child and mother- four measures describing the lexical and grammatical properties of CL and CDS (see Methods for more details on these measures and their computation). The richness of the vocabularies was measured using the lexical diversity measure ${ }^{29}$. The richness of the inflectional morphology paradigms was indexed by the inflectional diversity ${ }^{29}$. The diversity of the syntactic constructions used was measured by the mean length of utterances $\left(\mathrm{MLU}^{30}\right.$; a standard measure that accurately indexes syntactic diversity ${ }^{29}$ ). Finally, the total number of words produced by the child and the mother were taken as a measure of their respective loquacities.

Fig. 1 plots the evolution of the four measures in CL and in CDS. On one hand, the loquacity, inflectional diversity, and MLU series evidence a roughly linear increase along a child's development, with their values towards the end of the interval approaching what was found for their mothers. On the other hand, the lexical diversity measure exhibits quite constant patterns across most children, with their values being pretty much indistinguishable from those observed for their mothers (which are, even if slightly so, consistently increasing along time). Two of the children (Ruth and Nick) seem to be experiencing their 'vocabulary burst' later than the rest did. In fact, the inflectional diversity curves for these two children only begin their linear increases after the children have experienced their vocabulary bursts (see Extended Data Fig. 1). A similar pattern is found in the MLU curves for those two children. Their syntactic development is delayed by their late vocabulary bursts. These two patterns suggest that the development of both grammatical components of language (inflectional morphology and syntax) depends on having attained a certain degree of vocabulary richness. However, plain examination of these curves is not explicit 
evidence for the reliability of these hypothesized causal relationships; they could just be statistical mirages. For assessing such causal relations, one needs to turn to mCCM.

Fig. 2 depicts the network of causal relations discovered using mCCM. Of the pervasive correlations between pairs of measures, only a few constitute significant causality. Firstly, as one would expect from a usage-based perspective ${ }^{24}$, the specific amount of speech (i.e., loquacity) that each mother directs to her child influences the development of all aspects of child language. Also, as predicted by lexically-based syntax ${ }^{20,21}$, vocabulary growth in CDS causes increased MLUs (i.e., grammatical generalizations) in CL. In parallel, as predicted by syntactic bootstrapping ${ }^{22}$, increases in the syntactic knowledge of the child (i.e., syntactic diversity of CL) and in the diversity of syntactic structures to which they are exposed (i.e., syntactic diversity of CDS), both cause increases in the lexical knowledge of the child (i.e., lexical diversity of CL). In line with usage-based theories ${ }^{4-6}$, the syntax (and all other aspects of the CL) learned by children, rather than being innate -and therefore constant across children- specifically reflects the input that each child receives through $\operatorname{CDS}^{11,23,24}$. Crucially, the key prediction of the fine-tuning hypothesis ${ }^{10}$ is also supported: The diversity of syntactic constructions used by mothers (i.e., syntactic diversity of CDS) is caused by the diversity of constructions used by their children (i.e., syntactic diversity of CL) and by the diversity of inflectional forms they use (i.e., inflectional diversity of CL). In other words, mothers fine-tune their CDS in response to the specific grammatical (inflectional and/or syntactic) constructions used by their children ${ }^{3,9-11}$, rather than just to their general level of cognitive development ${ }^{8,12-14}$. Importantly however, mothers' fine-tuning is sensitive only to the grammatical structure in CL, inflectional morphology and syntax. This is a good choice, consid- 
ering that acquisition of grammar already entails previous advances in lexical. Furthermore, the causal relations found from the CL syntactic diversity to CDS syntactic diversity, and from the latter to all other aspects of CDS, ensures that the syntax of CL indirectly influences all aspects of CDS. In summary, our causality analyses match the predictions of usage-based theories of language acquisition ${ }^{4-6}$ better than those of nativist theories ${ }^{7,8}$ : Grammar is progressively generalized from the input, the language acquired depends on the input received, and mothers fine-tune their child-directed speech in response to the specific properties of child language.

1. Snow, C. E. Mothers' speech to children learning language. Child Dev. 43, 549-565 (1972).

2. Cross, T. G. Mothers' speech adjustments: The contribution of selected child listener variables. In Snow, C. E. \& Ferguson, C. A. (eds.) Talking to Children, 151-188 (Cambridge University Press, Cambridge, England, 1977).

3. Snow, C. E. Understanding social interaction and language acquisition; sentences are not enough. In Bornstein, M. H. \& Bruner, J. S. (eds.) Interaction in human development, Crosscurrents in contemporary psychology, 83-103 (Lawrence Erlbaum Associates, Hillsdale, NJ, 1989).

4. Tomasello, M. Beyond formalities: The case of language acquisition. Ling. Rev. 22, 183-197 (2005). 
5. Lieven, E. First language development: a usage-based perspective on past and current research. J. Child Lang. 41, 48-63 (2014).

6. MacWhinney, B. What we have learned. J. Child Lang. 41, 124-131 (2014).

7. Pinker, S. The language instinct: How the mind creates language (Harper-Collins, New York, NY, 1994).

8. Valian, V. Arguing about innateness. J. Child Lang. 41, 78-92 (2014).

9. Murray, A., Johnson, J. \& Peters, J. Fine-tuning of utterance length to preverbal infants: effects on later language development. J. Child Lang. 17, 511-525 (1990).

10. Sokolov, J. L. A local contingency analysis of the fine-tuning hypothesis. Dev. Psychol. 29, $1008-1023$ (1993).

11. Huttenlocher, J., Vasilyeva, M., Cymerman, E. \& Levine, S. Language input and child syntax. Cognit. Psychol. 45, 337-374 (2002).

12. Newport, E., Gleitman, H. \& Gleitman, L. Mother, I'd rather do it myself: Some effects and non-effects of maternal speech style. In Snow, C. E. \& Ferguson, C. A. (eds.) Talking to Children, 109-149 (Cambridge University Press, Cambridge, England, 1977).

13. Scarborough, H. \& Wycoff, J. Mother, I'd still do it myself: Some further non-effects of 'motherese'. J. Child Lang. 13, 431-437 (1986).

14. Valian, V. Input and language acquisition. In Ritchie, W. C. \& Bhattia, T. K. (eds.) Handbook of child language acquisition, 497-530 (Academic Press, San Diego, CA, 1999). 
15. van Geert, P. L. C. A dynamic systems model of cognitive and language growth. Psychol. Rev. 98, 3-53 (1991).

16. Dale, R. \& Spivey, M. J. Unraveling the dyad: Using recurrence analysis to explore patterns of syntactic coordination between children and caregivers in conversation. Lang. Learn. 391-430 (2006).

17. Steenbeek, H. W. \& van Geert, P. L. C. A theory and dynamic model of dyadic interaction: Concerns, appraisals, and contagiousness in a developmental context. Dev. Rev. 27, 1-40 (2007).

18. Sugihara, G. et al. Detecting causality in complex ecosystems. Science 338, 496-500 (2012).

19. Clark, A. T. et al. Spatial convergent cross mapping to detect causal relationships from short time series. Ecol. 96, 1174-1181 (2015).

20. Bates, E. \& Goodman, J. On the emergence of grammar from the lexicon. In MacWhiney, B. (ed.) The emergence of language, 29-80 (Lawrence Erlbaum Associates, Mahwah, NJ, 1999).

21. Lieven, E. V. M., Pine, J. M. \& Baldwin, G. Lexically-based learning and early grammatical development. J Child Lang. 24, 187-219 (1997).

22. Gleitman, L. R. The structural sources of verb meanings. Lang. Acq. 1, 3-55 (1990).

23. Hurtado, N., Marchman, V. A. \& Fernald, A. Does input influence uptake? Links between maternal talk processing speed and vocabulary size in Spanish-learning children. Dev. Sci. 11, F31-F39 (2008). 
24. Weisleder, A. \& Fernald, A. Talking to children matters: Early language experience strengthens processing and builds vocabulary. Psychol. Sci. 24, 2143-2152 (2013).

25. Takens, F. Detecting strange attractors in turbulence. In Rand, D. A. \& Young, L.-S. (eds.) Dynamical Systems and Turbulence, 366-381 (Springer Verlag, Berlin, Germany, 1981).

26. Abarbanel, H. D. I., Brown, R., Sidorowich, J. J. \& Tsimring, L. Sh. The analysis of observed chaotic data in physical systems. Rev. Mod. Phys. 65, 1331-1392 (1993).

27. Mønster, D., Fusarolli, R., Tylén, K., Roepstorff, A. \& Sherson, J. F. Inferring causality from noisy time series data: A test of Convergent Cross-Mapping. Futur. Gener. Comput. Syst. [published online] (2016).

28. Theakston, A. L., Lieven, E. V. M., Pine, J. M. \& Rowland, C. F. The role of performance limitations in the acquisition of verb-argument structure: an alternative account. J. Child Lang. 28, 127-152 (2001).

29. Moscoso del Prado Martín, F. Vocabulary, grammar, sex, and aging. Cognit. Sci. [published online] (2016).

30. Brown, R. A first language: the early stages (Harvard University Press, Cambridge, MA., 1973).

\section{Methods}

Materials We obtained the transcribed conversations from the Manchester Corpus $^{28}$, available in the CHILDES database ${ }^{31}$. This corpus contains annotated transcripts of audio recordings from a 
longitudinal study of 12 British English-speaking children ( 6 girls and 6 boys) between the ages of approximately two and three years. The children were recorded at their homes for an hour while they engaged in normal play activities with their mothers. Each child was recorded on two separate occasions in every three-week period for one year. Each recording session is divided into two halfhour periods. The annotations include the lemmatized form of the words produced by the children (incomplete words and small word-internal errors were manually corrected in the lemmatization).

Measures of Linguistic Development The amount of speech produced by a child or mother in a temporal window was measured by counting the total number of word tokens he/she produced in that period. We refer to this measure as the loquacity. The diversity of the different words used was measured by the lexical diversity ${ }^{29}$. This is the information entropy ${ }^{32}$ of the probability distribution of word lemmas found in the sample,

$$
H[L]=\sum_{\ell \in L} \mathrm{p}(\ell) \cdot \log \frac{1}{\mathrm{p}(\ell)},
$$

where $L$ refers to the set of word lemmas found in a sample, and $\mathrm{p}(\ell)$ is the probability with which the particular lemma $\ell$ is found in that sample. The richness of inflectional morphological paradigms was indexed by their inflectional diversity ${ }^{29}$ measure. This is the conditional entropy ${ }^{32}$ of the distribution of inflected word forms given the lemmas.

$$
H[W \mid L]=H[W, L]-H[L]
$$

where $H[L]$ is the lexical diversity measure described above, and $H[W, L]$ is the joint entropy between the inflected word forms and their corresponding lemmas,

$$
H[W, L]=\sum_{\ell \in L} \sum_{w \in W} \mathrm{p}(w, \ell) \cdot \log \frac{1}{\mathrm{p}(w, \ell)},
$$


where $L$ denotes the set of all distinct lemmas encountered in the sample, $W$ is the set of all distinct inflected word forms encountered, and $\mathrm{p}(w, \ell)$ is the joint probability with which lemma $\ell$ occurs as the specific inflected form $w$. Inflectional diversity takes non-negative values, measuring how large are the average inflectional paradigms used in the language sample ${ }^{29}$. Finally, following the standard in this field, the diversity of the syntactic structures used by the children and mothers was measured using the word-based ${ }^{33}$ mean length of utterances (MLU) ${ }^{30}$. In the ages studied, MLUs are well known to provide an accurate measure of the syntactic richness of the utterances produced ${ }^{30}$, and in fact correlate almost perfectly with explicit measurements of grammatical diversity $^{29}$.

Bias-Adjusted Entropy Estimation Entropy estimates obtained using Eq. 1 and Eq. 3 with maximum likelihood estimates of the probabilities are known to be strongly biased ${ }^{34}$, with the bias magnitude correlating with the size of the sample used. Importantly, the sample size is nothing else than the loquacity measure described above. Therefore, using this plain maximum likelihood method would result in spurious correlations. For this reason, it is more adequate ${ }^{29}$ to use the biasadjusted entropy estimator ${ }^{35}$ instead of Eq. 1 and Eq. 3. This is a very accurate estimator that relies on properties of the accumulation curve of the number of distinct words observed. The estimator is given by

$$
\hat{H}=\sum_{1 \leq F_{i} \leq n-1}\left[\frac{F_{i}}{n}\left(\sum_{k=F_{i}}^{n-1} \frac{1}{k}\right)\right]-\frac{f_{1}}{n}(1-A)^{1-n}\left[\log (A)+\sum_{r=1}^{n-1} \frac{1}{r}(1-A)^{r}\right]
$$


where $F_{i}$ are the word frequencies observed in the sample, $n$ is the number of tokens in the corpus, and

$$
A= \begin{cases}\frac{2 f_{2}}{(n-1) f_{1}+2 f_{2}} & \text { if } f_{2}>0, \\ \frac{2}{(n-1)\left(f_{1}-1\right)+2} & \text { if } f_{2}=0, f_{1}>0, \\ 1 & \text { if } f_{1}=f_{2}=0,\end{cases}
$$

with $f_{1}$ and $f_{2}$ being the number of word types that were encountered exactly once or twice respectively (i.e., the numbers of hapax legomena and dis legomena).

Reconstruction of Shadow Manifolds In order to increase the sample size in each period, we followed a sliding window technique: We computed measures for the samples contained in overlapping windows of three consecutive corpus files. In this way, at each point we obtained samples originating from two files from the same recording session, and a file from either the previous or the next recording session. Using this technique, for each child we obtained eight time series, two (one for CL and one for CDS) corresponding to each of the four measures described above: loquacity, lexical diversity, inflectional diversity, and MLU. The series were checked to ensure that they contained non-linear signal not dominated by noise. This was achieved using a prediction test ${ }^{19}$ : We ensured that, for all four variables, the ability to predict future values significantly decreased as one increases the distance in the future at which the predictions are being made (see Extended Data Fig. 3).

To provide further evidence for the presence of a non-linear deterministic component in the series, we used the method of sequential locally weighted global linear maps (S-map) ${ }^{36}$. This method relies on estimating a global fit (instead of the local fits used by the plain Simplex Projection ${ }^{37}$ 
method used in the previous tests) with a non-linearity parameter $\theta$ that increases the importance of local points in the prediction. In series having a non-linear deterministic component, plotting the prediction accuracy against this parameter results in curves peaking at a value $\theta>0$. Each of the sequences was standardized to zero mean and unit variance within each child, and detrended using a linear regression (to satisfy the stationarity requirement of the S-map method ${ }^{36}$ ). We applied the S-map on the linear regression residuals, which revealed the peaked curves that one would expect from series containing non-linear deterministic signal (see Extended Data Fig. 4). This indicates that that the non-linear deterministic component of the signal was sufficiently strong to remain detectable, even after removing the endogenous linear trend. Notice that this linear trend reflects the actual growths in CL and CDS, therefore the non-linear dynamics in this system extend beyond simple growth.

We reconstructed the shadow attractors from each of the collections of time series. The optimal time-lags $(\tau)$ for constructing the shadow manifolds were estimated as the first local minimum of the lagged self-information in each of the time $\operatorname{series}^{26}$. The optimal embedding dimensionalities $(E)$ were estimated by optimizing next-step prediction accuracy ${ }^{19}$ (see Figure 2 in the extended data). For each measure, we used a single pair of $(\tau, E)$ estimates for all children. The estimated parameter values used for the reconstruction of each shadow attractor are given in Table 1.

Detection of Causal Relationships Following the mCCM methodology ${ }^{19}$, we performed 5,000 bootstrapping iterations for assessing the $p$-values of the relations. In each bootstrap iteration, for every causal pair to be tested, we randomly selected twelve pairs of series (each pair coming from the same child/mother coupling, but the couplings themselves being selected with replacement 
from among all pairs). For each bootstrapping instance, we used leave-one-out cross-validation for assessing how well the series from one variable could be reconstructed from the other's shadow attractor using Simplex Projection ${ }^{37}$. This forecasting technique focuses on the system's dynamics, rather than on the measurement noise that will be present. The quality of the reconstruction was indexed by the Pearson correlation coefficient $(\rho[L])$ between the predicted and actual values. For each pair, these coefficients were computed for predictions with library sizes $(L)$ ranging from $L_{0}=\tau(E-1)+E+1$ points, to $L_{f}=\|x\|-E+2$ (where $\|x\|$ is the length of the predicting series) in increases of 40 points (the results are illustrated in Extended Data Fig. 5). The $p$ values were estimated by the proportion of bootstrap runs in which $\rho\left[L_{0}\right]<\rho\left[L_{f}\right]>0$. This is the number of bootstrapping runs in which the cross-mapping showed increased convergence with increasing library size, the hallmark of causality in $\mathrm{CCM}^{18,19}$. These raw $p$-values are provided in Extended Data Table 1.

Finally, to account for the very large number of tests performed (56 tests), with relatively vague a priori predictions, the raw $p$-values were adjusted for multiple comparisons using the false discovery rate (FDR) procedure for correlated test ${ }^{38}$. To remain conservative, in those cases where a relation appeared in all 5,000 bootstrapped or randomized trials, it was taken to have a $p$-value of $1 /(5000+1)=.00019996$ instead of .0000 (assuming uncorrected $p$-values of zero would mislead the FDR algorithm into too lax corrections). The resulting FDR-corrected $p$-values are provided in Extended Data Table 2.

Decoupling Analysis The possibility remains that the relationships found above reflect 'universal' developmental patterns (as one would predict from a strictly nativist perspective), as opposed to 
specific mother-child couplings (as would be expected by usage-based theories). If this were the case, one should be able to exchange series across mother-child pairs without having much effect on the causal relations. To investigate this possibility, we performed a decoupling analysis. For each of the 5,000 bootstrapping runs above, we generated a random sample, pairing each series from a child-mother dyad with one from a different dyad (while ensuring that all series were used in all tests, and that no series was used twice in the same set). We repeated the causality tests on these decoupled series. With decoupling, only two causal arrows were reliable, and both of them were also reliable in the original coupled analysis (which revealed twenty causal relations; for comparison see Extended Data Tables 1 and 2), indicating that the causal connections are substantially more reliable when pairing children with their actual mothers (see the clear difference in the spread of the curves in Extended Data Fig. 5). In addition, comparison of the amount of convergence (i.e., $\rho\left[L_{f}\right]-\rho\left[L_{0}\right]$ ) revealed that, beyond being more reliable, the convergence was significantly stronger for the coupled than it was for the decoupled pairs $(t[280000]=7.48, p<$ .0001, paired two-tailed).

Code Availability Custom Python code for computing the bias-adjusted entropy estimates of Eq. 4 can be obtained from the corresponding author. All mCCM computations were done using the implementation publicly available in the R software package multispatial $\mathrm{CCM}^{19}$.

\section{Additional (Methods-only) References}

31. MacWhinney, B. The CHILDES Project: Tools for analyzing talk, vol. 2: The database (Lawrence Erlbaum Associates, Mahwah, NJ, 2000), 3rd edn. 
32. Shannon, C. E. A mathematical theory of communication. Bell Syst. Tech. J. 27, 379-423, 623-656 (1948).

33. Parker, M. D. \& Brorson, K. A comparative study between mean length of utterance in morphemes (MLUm) and mean length of utterance in words (MLUw). First Lang. 25, 365-376 (2005).

34. Miller, G. Note on the bias of information estimates. In Quastler, H. (ed.) Information Theory in Psychology: Problems and Methods, 95-100 (Free Press, Glencoe, IL, 1955).

35. Chao, A., Wang, Y. T. \& Jost, L. Entropy and the species accumulation curve: a novel entropy estimator via discovery rates of new species. Methods Ecol. Evol. 4, 1091-1100 (2013).

36. Sugihara, G. Nonlinear forecasting for the classification of natural time series. Phil. Trans. $R$. Soc. Lond. A 348, 477-495 (1994).

37. Sugihara, G. \& May, R. M. Nonlinear forecasting as a way of distinguishing chaos from measurement error in time series. Nature 344, 734-741 (1990).

38. Benjamini, Y. \& Yekutieli, D. The control of the false discovery rate in multiple testing under dependency. Ann. Stat. 29, 1165-1188 (2001).

Acknowledgements The authors are indebted to John W. Du Bois and Laurie B. Feldman for useful suggestions on an earlier draft.

Competing Interests The authors declare that they have no competing financial interests. 
Author Contributions F.M.P.M. conceived and designed the study and wrote the paper. F.M.P.M., J.I. and D.S. performed the statistical analyses.

Correspondence Correspondence and requests for materials should be addressed to F.M.P.M. (email: fmoscoso@linguistics.ucsb.edu). 
Figure 1 Evolution of the measures studied as a function of the children's ages. The solid lines plot the evolution of the measures in CL and the dashed lines their evolution in CDS. The colors distinguish the individual child-mother dyads. To facilitate comparisons, all measures have been normalized to a $0-1$ scale.

Figure 2 Reconstructed network of causal relations recovered by mCCM. The shadowed boxes denote the CL measures, and the rounded boxes are the equivalent CDS measures. The double arrows highlight the causal relations from CL to CDS, which are evidence for fine-tuning. The dashed arrows mark the causal relations that were significant in both the coupled and decoupled analyses, all remaining arrows were significant in the coupled analysis alone. 


\begin{tabular}{cccccc}
\hline & Parameter & Loquacity & Lexical & Inflectional & DLU \\
Diversity & Diversity & \\
\hline Child Language & $\tau$ & 3 & 2 & 3 & 3 \\
\hline Child-Directed Speech & $E$ & 3 & 3 & 2 & 4 \\
\hline & $\tau$ & 3 & 3 & 2 & 3 \\
\hline
\end{tabular}

Table 1: (Methods) Parameter values used for shadow attractor reconstruction. 


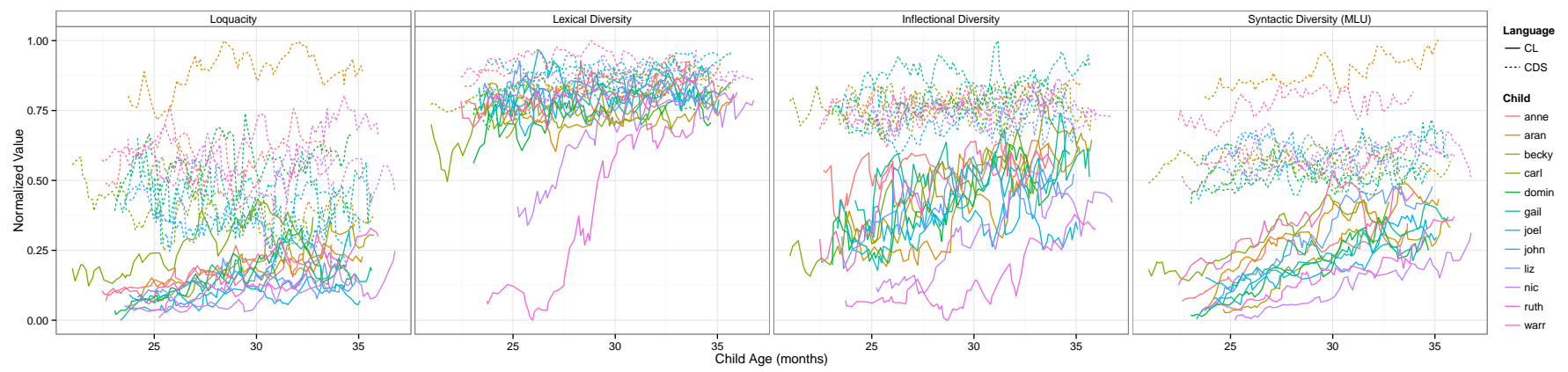

Figure 1. Evolution of the four measures studied as a function of the children's ages. The solid lines plot the evolution of the measures in $\mathrm{CL}$ and the dashed lines their evolution in CDS. The colors distinguish the child-mother dyads. For comparison, all four measures have been normalized to a $0-1$ scale. 


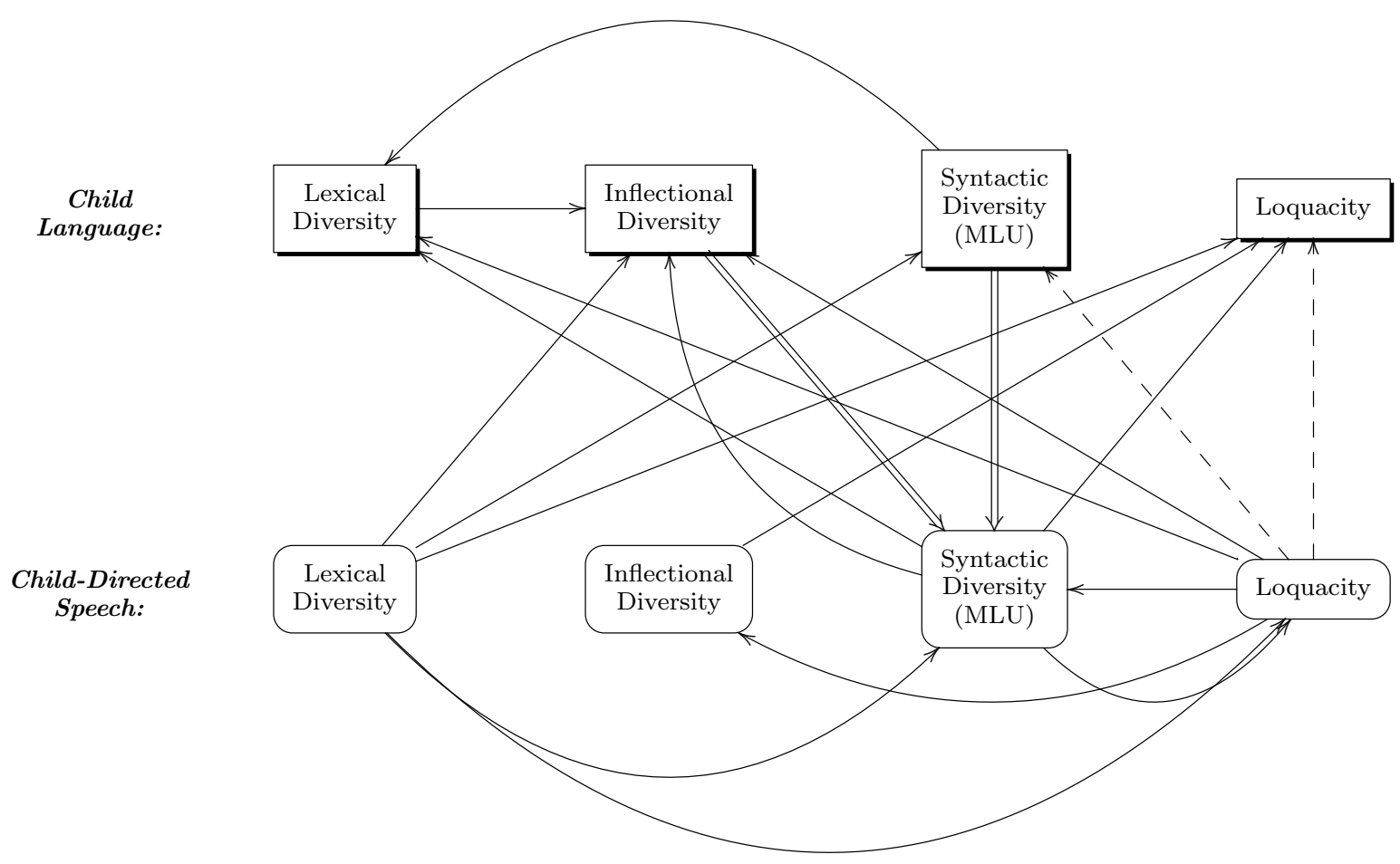

Figure 2. Reconstructed network of causal relations recovered by mCCM. The shadowed boxes denote the CL measures, and the rounded boxes are the equivalent CDS measures. The double arrows highlight the causal relations from CL to CDS, which are evidence for fine-tuning. The dashed arrows mark the causal relations that were significant in both the coupled and decoupled analyses, all remaining arrows were significant in the coupled analysis alone. 
- Child Language — Child Directed Speech
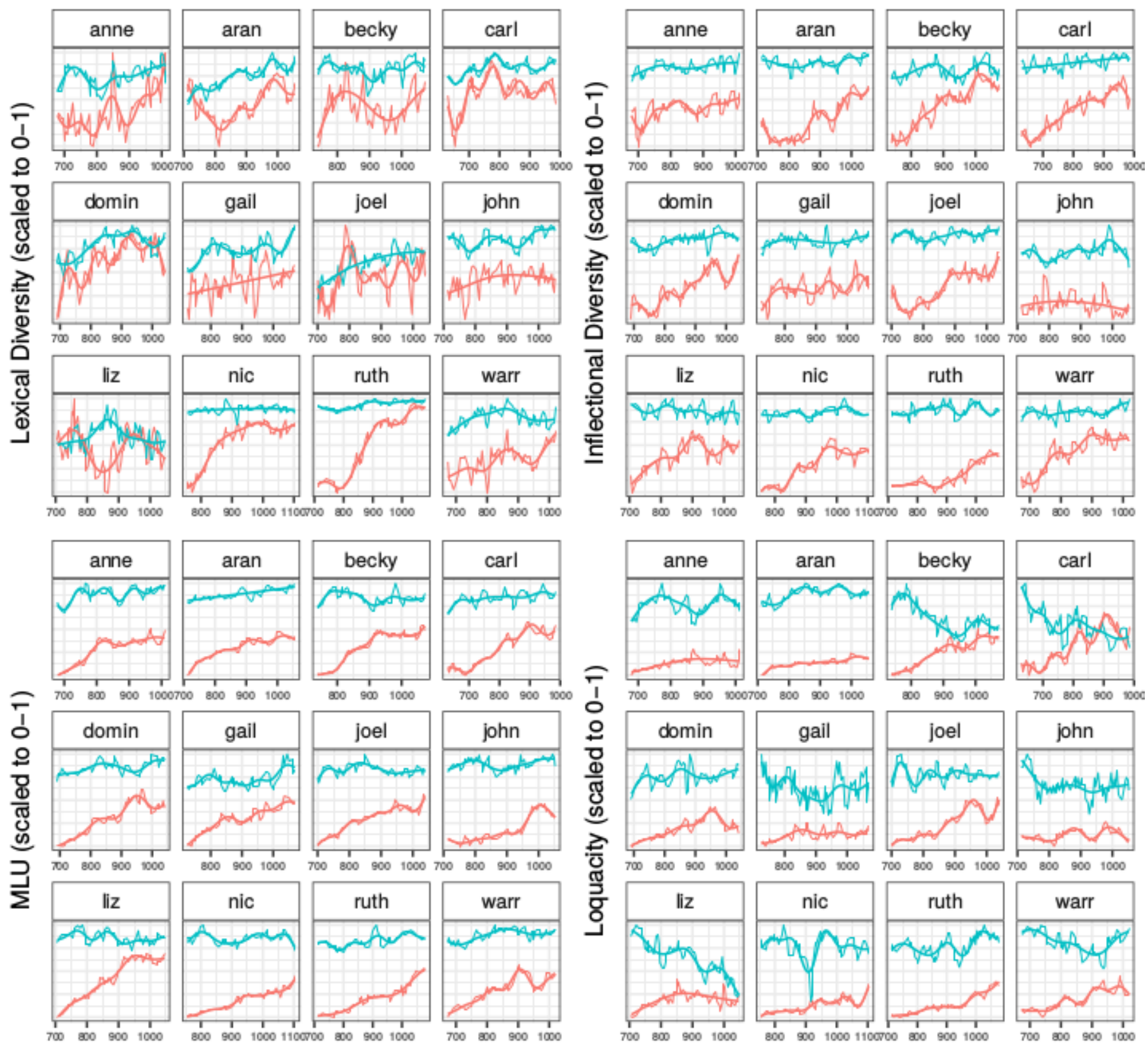
Age (days)

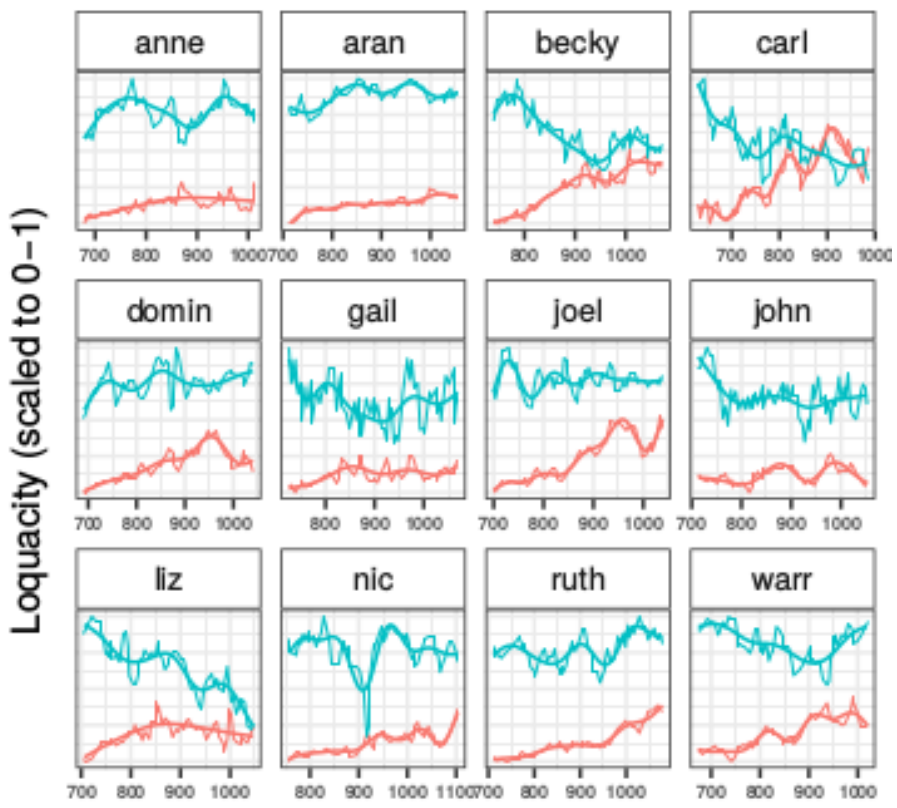

Age (days)

Extended Data Figure 1. Detailed evolution of the four measures in each child-mother pair. To facilitate comparing the patterns across mother-child pairs, all measures have been rescaled into $[0,1]$ within each panel (use Fig. 1 for comparing magnitudes across children or mothers). The thinner lines plot the raw measurements, and the thicker lines are restricted cubic spline smoothers (fitted using generalized additive models) highlighting the main trends for each child and mother. Blue lines plot measurements and smoothers on the mothers' speech, and red lines plot the equivalent measures on the children's speech. 

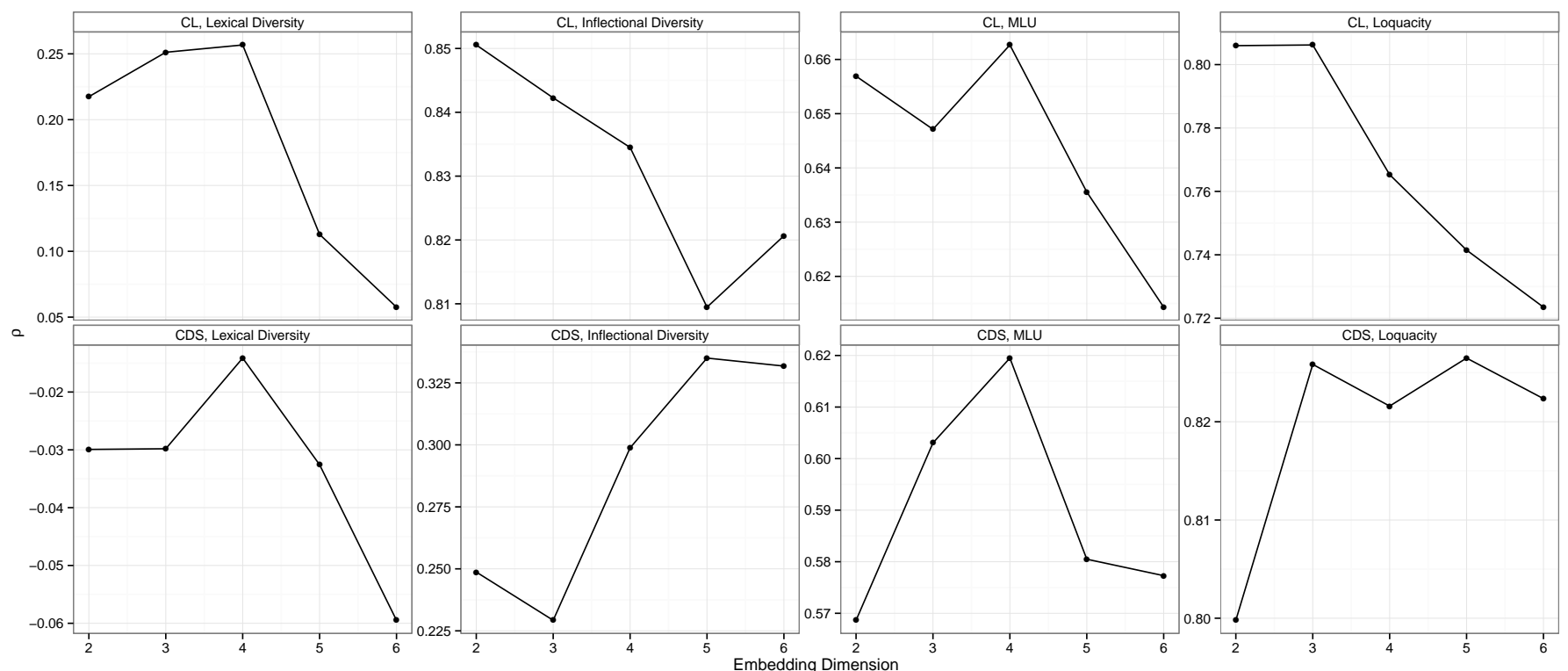

Extended Data Figure 2. Prediction tests used for selecting the optimal embedding dimension. The dimension is chosen for maximizing the next step prediction ability of the attractor ${ }^{19,26}$. The horizontal axes plot the candidate embedding dimensions, and the vertical axes plot the correlation coefficient $(\rho)$ between the true and predicted next points. 


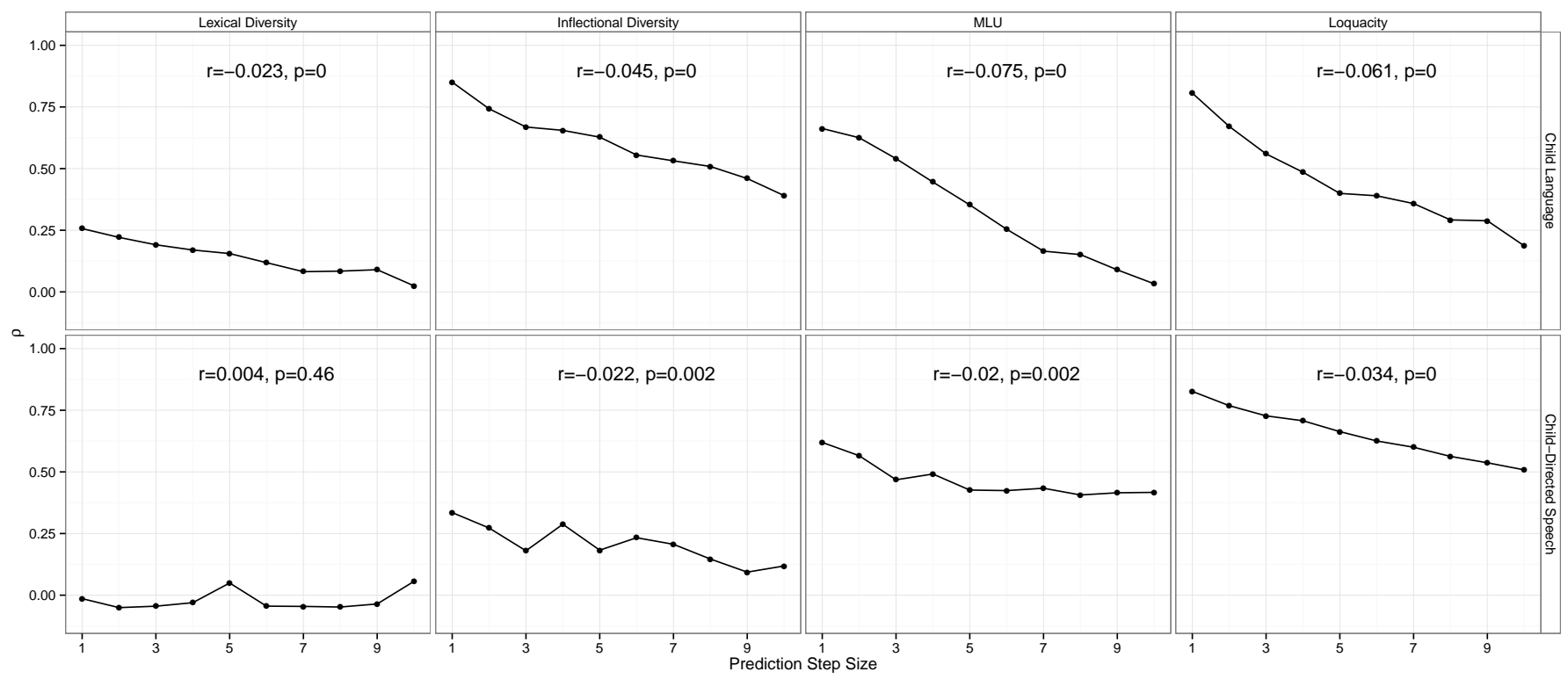

Extended Data Figure 3. Prediction tests used for assessing whether the series contained nonlinear dynamics. This is reflected in the negative correlations between the prediction quality based on the candidate reconstructed attractor $(\rho)$ and the distance at which the prediction is made, indicating that the signals are increasingly unpredictable. The graphs provide significant evidence (i.e., negative correlations) for the presence of nonlinear signal in all series but the Lexical Diversity in CDS. Notice that this is due to a floor effect, as in these series the predicted values are already uncorrelated with the true values at the first time step. 


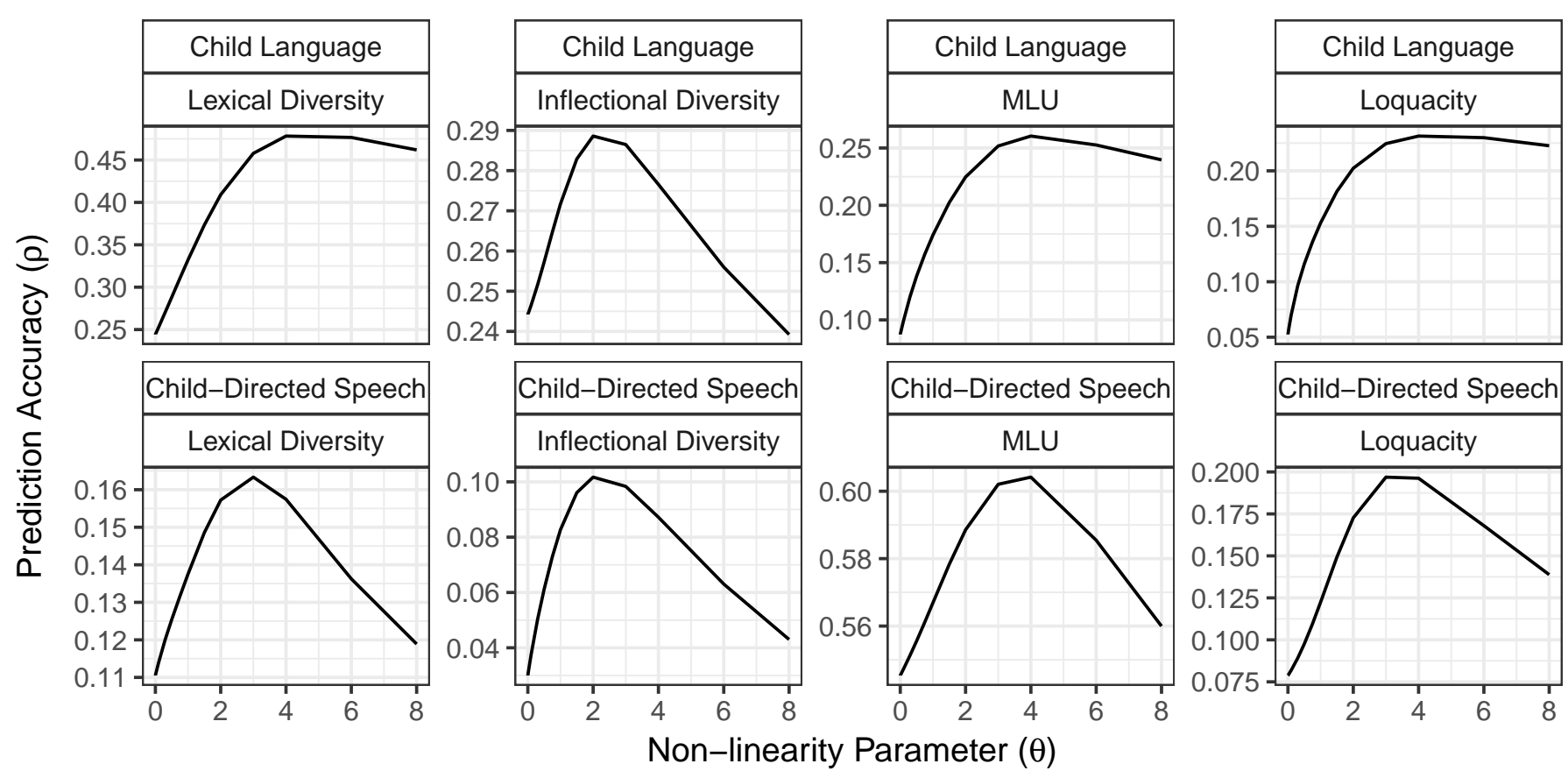

Extended Data Figure 4. S-map results (on linearly detrended series) plotting the prediction accuracy $(\rho)$ against the non-linearity parameter $\theta$. Note that all time series suggest nonlinear dynamics in the data, evidenced by the initial rise in $\rho$ for $\theta>0$, followed by a marked drop in $\rho$ with $\theta$. 
Library Size smallest largest Significance coupled both

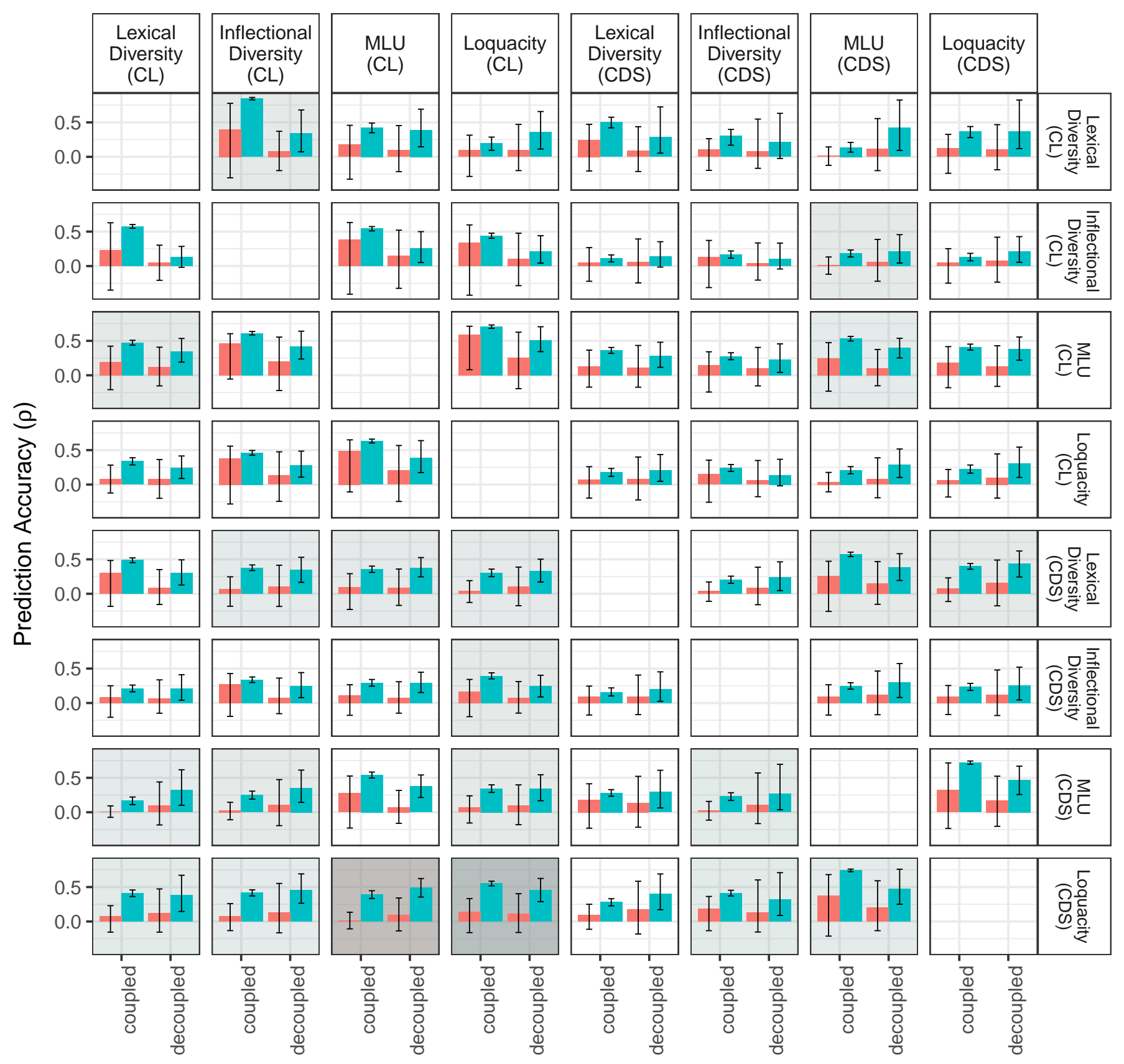

Extended Data Figure 5. Convergence of the series reconstruction accuracy (measured by the mean Pearson correlation coefficient $\rho$ ) for the smallest and largest library sizes. The error bars plot the interval for $95 \%$ of the bootstrapped simulations. The panels highlighted in light grey are those that reached significance in the coupled analysis only, and those shaded in a darker shade of grey are the ones that where significant in both coupled and decoupled analyses. Notice that the decoupled analysis results show more overlap between smallest and largest library sizes than the coupled analysis. This reflects how more of the coupled analyses exhibitted convergence. Causality arrows go from rows to columns. 


\begin{tabular}{|c|c|c|c|c|c|c|c|c|}
\hline $\begin{array}{c}\text { TO } \Rightarrow \\
\text { FROM } \Downarrow\end{array}$ & $\begin{array}{c}\text { Lexical } \\
\text { Diversity } \\
(\mathrm{CL}) \\
\end{array}$ & $\begin{array}{c}\text { Inflectional } \\
\text { Diversity } \\
(\mathrm{CL})\end{array}$ & $\begin{array}{l}\text { MLU } \\
(\mathrm{CL})\end{array}$ & $\begin{array}{l}\text { Loquacity } \\
\text { (CL) }\end{array}$ & $\begin{array}{c}\text { Lexical } \\
\text { Diversity } \\
(\mathrm{CDS})\end{array}$ & $\begin{array}{c}\text { Inflectional } \\
\text { Diversity } \\
\text { (CDS) }\end{array}$ & $\begin{array}{c}\text { MLU } \\
(\mathrm{CDS})\end{array}$ & $\begin{array}{l}\text { Loquacity } \\
\text { (CDS) }\end{array}$ \\
\hline $\begin{array}{c}\text { Lexical } \\
\text { Diversity } \\
(\mathrm{CL})\end{array}$ & & $\begin{array}{c}.0002 / \\
.0708\end{array}$ & $\begin{array}{c}.1246 / \\
.0196\end{array}$ & $\begin{array}{c}.4050 / \\
.0318\end{array}$ & $\begin{array}{c}.0188 / \\
.1276\end{array}$ & $\begin{array}{c}.0364 / \\
.2250\end{array}$ & $\begin{array}{c}.0642 / \\
.0550\end{array}$ & $\begin{array}{c}.0158 / \\
.0482\end{array}$ \\
\hline $\begin{array}{c}\text { Inflectional } \\
\text { Diversity } \\
\text { (CL) }\end{array}$ & $\begin{array}{c}.0838 / \\
.2874\end{array}$ & & $\begin{array}{c}.3602 / \\
.4028\end{array}$ & $\begin{array}{c}.5062 / \\
.3840\end{array}$ & $\begin{array}{c}.4540 / \\
.3548\end{array}$ & $\begin{array}{c}.5556 / \\
.3822\end{array}$ & $\begin{array}{c}.0032 / \\
.1906\end{array}$ & $\begin{array}{c}.4034 / \\
.1962\end{array}$ \\
\hline $\begin{array}{l}\text { MLU } \\
(\mathrm{CL}) \\
\end{array}$ & $\begin{array}{c}.0022 / \\
.0234 \\
\end{array}$ & $\begin{array}{c}.0206 / \\
.0342 \\
\end{array}$ & & $\begin{array}{c}.0500 / \\
.0150 \\
\end{array}$ & $\begin{array}{c}.0304 / \\
.0886 \\
\end{array}$ & $\begin{array}{c}.2224 / \\
.1618 \\
\end{array}$ & $\begin{array}{c}<.0002 / \\
.0016 \\
\end{array}$ & $\begin{array}{c}.0454 / \\
.0106 \\
\end{array}$ \\
\hline $\begin{array}{c}\text { Loquacity } \\
\text { (CL) }\end{array}$ & $\begin{array}{c}.0054 / \\
.1162\end{array}$ & $\begin{array}{c}.5026 / \\
.2232\end{array}$ & $\begin{array}{c}.0916 / \\
.1162\end{array}$ & & $\begin{array}{c}.2456 / \\
.2064\end{array}$ & $\begin{array}{c}.3820 / \\
.2842\end{array}$ & $\begin{array}{c}.0106 / \\
.0596\end{array}$ & $\begin{array}{c}.0352 / \\
.0812\end{array}$ \\
\hline $\begin{array}{l}\text { Lexical } \\
\text { Diversity } \\
(\mathrm{CDS})\end{array}$ & $\begin{array}{c}.0290 / \\
.0180\end{array}$ & $\begin{array}{c}.0002 / \\
.0214\end{array}$ & $\begin{array}{c}.0008 / \\
.0012\end{array}$ & $\begin{array}{c}.0002 / \\
.0222\end{array}$ & & $\begin{array}{c}.0102 / \\
.1090\end{array}$ & $\begin{array}{c}<.0002 / \\
.0262\end{array}$ & $\begin{array}{c}<.0002 / \\
.0104\end{array}$ \\
\hline $\begin{array}{c}\text { Inflectional } \\
\text { Diversity } \\
\text { (CDS) }\end{array}$ & $\begin{array}{c}.1690 / \\
.1036\end{array}$ & $\begin{array}{c}.4660 / \\
.0768\end{array}$ & $\begin{array}{c}.0136 / \\
.0074\end{array}$ & $\begin{array}{c}.0012 / \\
.0520\end{array}$ & $\begin{array}{c}.3344 / \\
.2150\end{array}$ & & $\begin{array}{c}.0662 / \\
.0868\end{array}$ & $\begin{array}{c}.0752 / \\
.1706\end{array}$ \\
\hline $\begin{array}{c}\text { MLU } \\
(\mathrm{CDS})\end{array}$ & $\begin{array}{c}.0006 / \\
.0400\end{array}$ & $\begin{array}{c}.0002 / \\
.0508\end{array}$ & $\begin{array}{c}.0200 / \\
.0016\end{array}$ & $\begin{array}{c}<.0002 / \\
.0184\end{array}$ & $\begin{array}{c}.3586 / \\
.1622\end{array}$ & $\begin{array}{c}.0004 / \\
.1222\end{array}$ & & $\begin{array}{c}.0222 / \\
.0086\end{array}$ \\
\hline $\begin{array}{l}\text { Loquacity } \\
\text { (CDS) }\end{array}$ & $\begin{array}{c}<.0002 / \\
.0138\end{array}$ & $\begin{array}{c}<.0002 / \\
.0026\end{array}$ & $\begin{array}{c}<.0002 / \\
<.0002\end{array}$ & $\begin{array}{c}<.0002 / \\
.0002\end{array}$ & $\begin{array}{c}.0100 / \\
.0336\end{array}$ & $\begin{array}{c}.0014 / \\
.0570\end{array}$ & $\begin{array}{c}<.0002 / \\
.0132\end{array}$ & \\
\hline
\end{tabular}

Extended Data Table 1. Raw mCCM p-values (i.e., prior to FDR correction) obtained from 5,000 bootstrapping runs for each pair of variables. The causal arrows tested go from rows to columns. Each cell contains two $p$-values, in the format $p$ (coupled) / $p$ (decoupled), where coupled refers to the original analysis, and decoupled to the one where the series were randomized across child-mother pairs. $p$-values with a value $<.0002$ refer to causal relations in which convergence was found in every single one of the 5,000 bootstrapped samples (i.e., technically $p \approx .0000$ ). 


\begin{tabular}{|c|c|c|c|c|c|c|c|c|}
\hline $\begin{array}{c}\text { TO } \Rightarrow \\
\text { FROM } \Downarrow\end{array}$ & $\begin{array}{c}\text { Lexical } \\
\text { Diversity } \\
(\mathrm{CL})\end{array}$ & $\begin{array}{c}\text { Inflectional } \\
\text { Diversity } \\
\text { (CL) }\end{array}$ & $\begin{array}{l}\text { MLU } \\
(\mathrm{CL})\end{array}$ & $\begin{array}{l}\text { Loquacity } \\
\text { (CL) }\end{array}$ & $\begin{array}{c}\text { Lexical } \\
\text { Diversity } \\
(\mathrm{CDS})\end{array}$ & $\begin{array}{c}\text { Inflectional } \\
\text { Diversity } \\
\text { (CDS) }\end{array}$ & $\begin{array}{c}\text { MLU } \\
(\mathrm{CDS})\end{array}$ & $\begin{array}{l}\text { Loquacity } \\
\text { (CDS) }\end{array}$ \\
\hline $\begin{array}{c}\text { Lexical } \\
\text { Diversity } \\
(\mathrm{CL})\end{array}$ & & $\begin{array}{c}.0040 / \\
.5898\end{array}$ & $\begin{array}{c}.7661 / \\
.3163\end{array}$ & $\begin{array}{c}1.000 / \\
.3840\end{array}$ & $\begin{array}{c}.1798 / \\
.8037\end{array}$ & $\begin{array}{c}.2765 / \\
1.000\end{array}$ & $\begin{array}{c}.4481 / \\
.5073\end{array}$ & $\begin{array}{c}.1569 / \\
.4974\end{array}$ \\
\hline $\begin{array}{l}\text { Inflectional } \\
\text { Diversity } \\
\text { (CL) }\end{array}$ & $\begin{array}{c}.5410 / \\
1.000\end{array}$ & & $\begin{array}{c}1.000 / \\
1.000\end{array}$ & $\begin{array}{c}1.000 / \\
1.000\end{array}$ & $\begin{array}{c}1.000 / \\
1.000\end{array}$ & $\begin{array}{c}1.000 / \\
1.000\end{array}$ & $\begin{array}{c}.0413 / \\
1.000\end{array}$ & $\begin{array}{c}1.000 / \\
1.000\end{array}$ \\
\hline $\begin{array}{l}\text { MLU } \\
(\mathrm{CL})\end{array}$ & $\begin{array}{c}.0299 / \\
.3180\end{array}$ & $\begin{array}{c}.1834 / \\
.3840\end{array}$ & & $\begin{array}{c}.3587 / \\
.2980\end{array}$ & $\begin{array}{c}.2453 / \\
.6537\end{array}$ & $\begin{array}{c}1.000 / \\
.9741\end{array}$ & $\begin{array}{c}.0040 / \\
.0826\end{array}$ & $\begin{array}{c}.3350 / \\
.2737\end{array}$ \\
\hline $\begin{array}{c}\text { Loquacity } \\
\text { (CL) }\end{array}$ & $\begin{array}{c}.0664 / \\
.7694\end{array}$ & $\begin{array}{c}1.000 / \\
1.000\end{array}$ & $\begin{array}{c}.5770 / \\
.7694\end{array}$ & & $\begin{array}{c}1.000 / \\
1.000\end{array}$ & $\begin{array}{c}1.000 / \\
1.000\end{array}$ & $\begin{array}{c}.1141 / \\
.5130\end{array}$ & $\begin{array}{c}.2755 / \\
.6354\end{array}$ \\
\hline $\begin{array}{c}\text { Lexical } \\
\text { Diversity } \\
(\mathrm{CDS})\end{array}$ & $\begin{array}{c}.2416 / \\
.3163\end{array}$ & $\begin{array}{c}.0040 / \\
.3180\end{array}$ & $\begin{array}{c}.0129 / \\
.0826\end{array}$ & $\begin{array}{c}.0040 / \\
.3180\end{array}$ & & $\begin{array}{c}.1141 / \\
.7608\end{array}$ & $\begin{array}{c}.0040 / \\
.3383\end{array}$ & $\begin{array}{c}.0040 / \\
.2737\end{array}$ \\
\hline $\begin{array}{l}\text { Inflectional } \\
\text { Diversity } \\
\text { (CDS) }\end{array}$ & $\begin{array}{c}1.000 / \\
.7432\end{array}$ & $\begin{array}{c}1.000 / \\
.6198\end{array}$ & $\begin{array}{c}.1405 / \\
.2730\end{array}$ & $\begin{array}{c}.0182 / \\
.4974\end{array}$ & $\begin{array}{c}1.000 / \\
1.000\end{array}$ & & $\begin{array}{c}.4499 / \\
.6537\end{array}$ & $\begin{array}{c}.4979 / \\
1.000\end{array}$ \\
\hline $\begin{array}{c}\text { MLU } \\
(\mathrm{CDS})\end{array}$ & $\begin{array}{c}.0103 / \\
.4304\end{array}$ & $\begin{array}{c}.0040 / \\
.4974\end{array}$ & $\begin{array}{c}.1834 / \\
.0826\end{array}$ & $\begin{array}{c}.0040 / \\
.3163\end{array}$ & $\begin{array}{c}1.000 / \\
.9741\end{array}$ & $\begin{array}{c}.0074 / \\
.7889\end{array}$ & & $\begin{array}{c}.1911 / \\
.2737\end{array}$ \\
\hline $\begin{array}{c}\text { Loquacity } \\
\text { (CDS) }\end{array}$ & $\begin{array}{c}.0040 / \\
.2970\end{array}$ & $\begin{array}{c}.0040 / \\
.1119\end{array}$ & $\begin{array}{c}.0040 / \\
.0258\end{array}$ & $\begin{array}{c}.0040 / \\
.0258\end{array}$ & $\begin{array}{c}.1141 / \\
.3840\end{array}$ & $\begin{array}{c}.0201 / \\
.5076\end{array}$ & $\begin{array}{c}. \mathbf{0 0 4 0} / \\
.2970\end{array}$ & \\
\hline
\end{tabular}

Extended Data Table 2. FDR-corrected mCCM $p$-values for each pair of variables. The causal arrows tested go from rows to columns. Each cell contains two $p$-values, in the format $p$ (coupled) / $p$ (decoupled), where coupled refers to the original analysis, and decoupled to the one where the series were randomized across child-mother pairs. The causal arrows that were significant at $\alpha=.05$ are highlighted in bold, and correspond to those arrows plotted in Fig. 1, and whose convergence plots are shown in Extended Data Fig. 4. 